\title{
Does the Use of Newer Technology to Divide the Mesoappendix Result in Less Bleeding? A Retrospective Analysis
}

\author{
Matthew P. Doepker ${ }^{a}$, Justin L. Gregg ${ }^{\mathrm{b}, \mathrm{d}}$, Giacomo Meeker ${ }^{\mathrm{a}}$, \\ Bryan Ellis ${ }^{\mathrm{c}}$, Kevin Grannan ${ }^{\mathrm{a}}$
}

\begin{abstract}
Background: Various methods have been used to seal and divide the mesoappendix, such as endoscopic linear cutting staples, the Harmonic Scalpel and even monopolar coagulation. The goal of this study was to determine whether using any of these energy sources versus any non-energy source would be superior in terms of post-operative complications (primarily bleeding), hospital length of stay and cost.
\end{abstract}

Methods: This study included all patients who underwent laparoscopic appendectomy for acute appendicitis between January 2007 and April 2010 at a community hospital system with two large hospitals. The primary outcomes measured were bleeding, postoperative anemia, blood transfusion requirements, length of stay and cost.

Results: Laparoscopic appendectomy was performed on 838 patients. An energy source was used on 503 patients and a non-energy source was used on 335 patients. No statistically significant differences were found regarding post-operative complications or length of stay. There was, however, a financial difference found, in that it was much less expensive to use a non-energy source versus an energy source when handling and controlling the mesoappendix.

Conclusions: Using a non-energy source is as safe as an energy source for dividing and sealing of the mesoappendix. This study supports the use of a non-energy source as a comparable method when compared to a non-energy source, with no difference in main outcomes.

\footnotetext{
Manuscript accepted for publication September 26, 2013

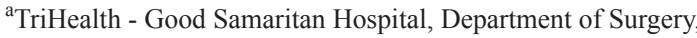
Cincinnati, Ohio, USA

${ }^{\mathrm{b}}$ TriHealth - E. Kenneth Hatton MD, Institute for Research and Education, Cincinnati, Ohio, USA

${ }^{\mathrm{c}}$ TriHealth - Bethesda North Hospital, Department of Surgery, Cincinnati, Ohio, USA

${ }^{\mathrm{d} C o r r e s p o n d i n g ~ a u t h o r: ~ J u s t i n ~ G r e g g, ~ T r i H e a l t h ~-~ H a t t o n ~ R e s e a r c h ~}$ Institute, 375 Dixmyth Avenue, Unit 11J, Cincinnati, Ohio 45220, USA. Email: justin_gregg@trihealth.com
}

doi: http://dx.doi.org/10.14740/jcs198w
Keywords: Laparoscopic appendectomy; Energy source; Non-energy source; Bleeding; Division of mesoappendix; Post-operative complications; Length of stay; Cost

\section{Introduction}

Appendectomy is one of the most common surgical procedures performed today. Since the introduction of laparoscopic appendectomy in 1983, the laparoscopic approach has surpassed the traditional open approach in frequency of usage. Meta-analyses of randomized-controlled trials have shown that laparoscopic appendectomy has several advantages over the open approach, including faster convalescence, less postoperative pain, better cosmetic results and fewer wound infections [1-3].

Prior studies have shown that the most common reason to convert to an open appendectomy is bleeding, specifically bleeding during the dissection and sealing of the mesoappendix [1-8]. As new technology emerges, it is imperative to examine and critically appraise new technology in terms of efficiency, quality and cost effectiveness. This study will examine and compare the potential differences between the use of energy and non-energy sources during laparoscopic appendectomy, in terms of intra-operative complications (for example, bleeding), post-operative course and cost.

\section{Materials and Methods}

After obtaining approval from the Institutional Review Board (IRB), patient data were retrospectively reviewed. Patients aged 18 years and older that underwent laparoscopic appendectomy for acute appendicitis at two community hospitals between January 1, 2007 and April 30, 2010 were included in this study. Exclusion criteria included patients who underwent laparoscopic appendectomy without pathologic confirmation of appendicitis, and patients who had additional surgeries at the time of their appendectomy, or if the operation was converted to open. Only one patient was converted to open and thus, excluded from the study. Of the 838 patients 
Table 1. Instruments Used and Quantity

\begin{tabular}{lll}
\hline Instrument & Patients $(\mathbf{n}=\mathbf{8 3 8})$ & $\mathbf{\%}$ \\
\hline LigaSure & 354 & $42.2 \%$ \\
Harmonic Scalpel & 118 & $14.0 \%$ \\
Endo GIA Stapler & 333 & $39.7 \%$ \\
Electrocautery \& Scissors & 32 & $3.8 \%$ \\
Endoloops & 1 & $0.1 \%$ \\
\hline
\end{tabular}

included in the study, 503 patients had their mesoappendix sealed with devices such as Ligasure, Harmonic Scalpel and monopolar electrocautery. This group was designated as the "energy source" group. Three hundred thirty-five (335) patients had their mesoappendix sealed with a non-energy source such as the Endo-GIA stapler, endoloops or endoclips. This group was designated as the "non-energy source" group. Patient outcomes were compared, which included bleeding, post-operative anemia, transfusion requirements and length of stay. Bleeding was defined as a decrease in hematocrit of ten or more percentage points, whereas postoperative anemia was defined only as a hematocrit of less than $30 \%$. A complete blood count was obtained at admission and on post-operative day number one the values were compared. The financial difference between the energy and non-energy sources was determined by using a range of costs of the various devices used in the hospital system.

The statistical analyses of the collected data were performed using an unpaired Student's $t$ test as well as a univariate chi-square test. Fisher's exact test was used for expected cell frequencies that were $<5$. The significance level for all analysis was $\alpha=0.05$. Statistical analyses were performed using SPSS 21.0 for Windows (SPSS, Chicago, IL, USA).

\section{Results}

The final analysis included 838 patients. A missing data pattern analysis was performed to determine if missing data would have any significant effects on study variables. It was determined that missing data points were missing completely at random and had no pertinent effects on the statistical analyses performed. The post-operative histopathologic diagnosis confirmed acute appendicitis in all patients. The total number of patients from one hospital was 577 and 261 patients were from the other hospital. The energy source group included 503 patients compared with 335 in the non-energy source. Both groups are summarized in Table 1.

Univariate chi-square analysis tested whether there was a statistically significant difference between the methods of division of the mesoappendix (energy vs. non-energy) with the development of surgical complications such as bleeding. Bleeding, as defined in the methods section, occurred in 43 patients $(5.1 \%)$ in the energy group compared to only 27 $(3.2 \%)$ in the non-energy group. Of the 43 patients who had bleeding events in the energy group, 32 patients $(3.8 \%)$ utilized the Ligasure, 8 patients $(0.9 \%)$ with Harmonic Scalpel and 3 patients $(0.3 \%)$ with electrocautery with scissors. All

Table 2. Perioperative Outcomes by Method of Division

\begin{tabular}{llll}
\hline Variable & Energy $\mathbf{n}=\mathbf{5 0 3} \mathbf{n}(\mathbf{\%})$ & Non-Energy $\mathbf{n}=\mathbf{3 3 5} \mathbf{n}(\mathbf{\%})$ & P value \\
\hline Post-Op Anemia & $25(4.9 \%)$ & $15(4.5 \%)$ & 0.345 \\
Post-Op Transfusion & $4(0.8 \%)$ & $1(0.3 \%)$ & 0.653 \\
Bleeding & $43(8.5 \%)$ & $27(8.1 \%)$ & 0.275 \\
LOS (Mean $\pm \mathrm{SD})$ & $1.65 \pm 1.73$ & $1.72 \pm 1.88$ & 0.618 \\
\hline
\end{tabular}

Post-op: post-operative; LOS: length of stay. 
Table 3. Cost

\begin{tabular}{ll} 
Instrument & Cost \\
\hline LigaSure + stapler & $\$ 1,744$ \\
Harmonic Scalpel + stapler & $\$ 1,450$ \\
Endo-GIA Stapler + reload & $\$ 699$ \\
Endoloops & $\$ 60$ \\
Electrocautery \& Scissors & $\$ 0$ \\
\hline
\end{tabular}

27 patients $(3.2 \%)$ in the non-energy group who experienced bleeding were in the Endo-GIA Stapler group. None of these differences were statistically significant using an omnibus test $(\mathrm{P}=0.548)$.

Four patients in the energy group required post-operative blood transfusions and 1 patient in the non-energy group required a blood transfusion. However, there was no statistical significant difference between the two groups ( $\mathrm{P}$ $=0.653)$. There were 25 patients in the energy group who experienced post-operative anemia compared to only 15 in the non-energy group. There was no statistical significance found regarding this variable $(\mathrm{P}=0.345)$. The mean length of stay (LOS) was 1.65 days in the energy source group and 1.72 days in the non-energy source group. This difference was not statistically significant $(\mathrm{P}=0.618)$ (Table 2$)$.

The financial difference of the various methods of sealing the mesoappendix was assessed by the cost of the devices to the hospital system with results summarized in Table 3. There was a substantial difference in cost between methods when comparing energy sources to non-energy sources. Virtually all surgeons included in this study routinely used an Endo-GIA to divide the base of the appendix, whether they were using an energy or a non-energy source to transect the mesoappendix; the mesoappendix was divided either with a vascular "reload" of the stapler, or an alternate energy source. The use of an energy source added approximately $\$ 700-1,000$ per case in cost to the hospitals for the devices.

\section{Discussion}

In our study, an energy source and a non-energy source were compared regarding the division and sealing of the mesoappendix. There were no significant differences in length of stay, post-operative complications such as bleeding, postoperative anemia or transfusion requirements. There was a substantial financial difference found between the different methods utilized. Although we did not attempt to show a statistical difference in cost, it seems clear that use of the non- energy methods to divide the mesoappendix is less expensive while achieving the same results, specifically hemostasis.

As with open appendectomy, the laparoscopic approach involves the dissection and division of the mesoappendix in order to seal and ligate the appendiceal artery. Historically, bleeding from the mesoappendix during dissection has been a reason for conversion to open [3]. When dealing with an extremely inflamed, gangrenous, or perforated appendix, using an energy source might intuitively seem to be advantageous; however, our data seem to demonstrate otherwise. The surgeon can use either of these energy sources to divide the mesoappendix without excessive dissection or isolation of the appendicular artery, leading to quicker division with minimal bleeding [4, 5, 9]. Using an Endo-GIA stapler, endoloops, or endoclips may require more meticulous dissection to safely isolate and divide the mesoappendix. This could theoretically lead to increased time and potentially more bleeding with subsequent anemia and the possible need for blood transfusion; however, our data refute this as well.

Although the literature has shown the utility of using energy sources, both the Endo-GIA stapling device and endoloops are valid and acceptable options $[4,5]$. When operating for early acute appendicitis, there is minimal inflammation and edema of the surrounding tissue, thus gaining control of the mesoappendix can be accomplished easily using endoloops or an Endo-GIA stapler with minimal manipulation of the tissues. This can result in fewer complications [6]. In addition, using either method can close the appendicular stump after the mesoappendix has been ligated without increase incidence of stump leakage [7, 9-11].

Like any study, this study faces limitations. One limitation was related to the study design. The retrospective nature of the study did not allow insight into the surgeons' discretion in using an energy source versus a non-energy source. More specifically, did the surgeon choose to use a certain method because of familiarity for the device, or was it due to clinical judgment based on the severity of the appendicitis? Although we did not look specifically at individual surgeons' variability in use of energy versus non-energy sources, it ap- 
peared that the use was not driven by clinical judgment during the cases. Individual surgeons either always used one of the devices, or never did.

Another limitation of the study was not examining the length of the procedures. Previous studies have shown that although using an energy source is more expensive, the amount of surgical time saved makes up for the difference [12]. This may be a direction for future research.

\section{Conclusion}

There was no difference in the development of post-operative hemorrhagic complications or length of stay based on the method of division of the mesoappendix using an energy source or a non-energy source. There was a financial difference between the two methods, with the use of an energy source to divide the mesoappendix being more expensive than using a non-energy source. As payments for healthcare shifts towards the "accountable care organization" model, surgeons and hospitals will need to seek the safest as well as the most cost-effective methods for patient care.

\section{Grant Support}

No grant support.

\section{Financial Disclosures}

No financial disclosures.

\section{References}

1. Ignacio RC, Burke R, Spencer D, Bissell C, Dorsainvil C, Lucha PA. Laparoscopic versus open appendectomy: what is the real difference? Results of a prospective randomized double-blinded trial. Surg Endosc. 2004;18(2):334-337.

2. Martin LC, Puente I, Sosa JL, Bassin A, Breslaw R, McKenney MG, Ginzburg E, et al. Open versus laparo- scopic appendectomy. A prospective randomized comparison. Ann Surg. 1995;222(3):256-261; discussion 261-252.

3. Katkhouda N, Mason RJ, Towfigh S, Gevorgyan A, Essani R. Laparoscopic versus open appendectomy: a prospective randomized double-blind study. Ann Surg. 2005;242(3):439-448; discussion 448-450.

4. Sucullu I, Filiz AI, Kurt Y, Yilmaz I, Yildiz M. The effects of LigaSure on the laparoscopic management of acute appendicitis: "LigaSure assisted laparoscopic appendectomy". Surg Laparosc Endosc Percutan Tech. 2009;19(4):333-335.

5. Aydogan F, Saribeyoglu K, Simsek O, Salihoglu Z, Carkman S, Salihoglu T, Karatas A, et al. Comparison of the electrothermal vessel-sealing system versus endoclip in laparoscopic appendectomy. J Laparoendosc Adv Surg Tech A. 2009;19(3):375-378.

6. Hanssen A, Plotnikov S, Dubois R. Laparoscopic appendectomy using a polymeric clip to close the appendicular stump. JSLS. 2007;11(1):59-62.

7. Martin del Olmo JC, Blanco Alvarez JI, Carbajo Caballero MA, de la Cuesta de la Llave C, Vaquero Puerta C, Arenal J. Laparoscopic appendectomy by ultrasonically activated scalpel in acute appendicitis: preliminary report. J Laparoendosc Adv Surg Tech A. 2002;12(2):111113.

8. Tonouchi H, Ohmori Y, Kobayashi M, Kusunoki M. Trocar site hernia. Arch Surg. 2004;139(11):1248-1256.

9. Schafer M, Lauper M, Krahenbuhl L. A nation's experience of bleeding complications during laparoscopy. Am J Surg. 2000;180(1):73-77.

10. Kazemier G, in't Hof KH, Saad S, Bonjer HJ, Sauerland S. Securing the appendiceal stump in laparoscopic appendectomy: evidence for routine stapling? Surg Endosc. 2006;20(9):1473-1476.

11. Yang H-R, Wang Y-C, Chung P-K, Jeng L-B, Chen R-J. Laparoscopic appendectomy using the ligasure vessel sealing system. J Laparoendosc Adv Surg Tech. 2005; 15(4):353-356.

12. McCahill LE, Pellegrini CA, Wiggins T, Helton WS. A clinical outcome and cost analysis of laparoscopic versus open appendectomy. Am J Surg. 1996;171(5):533537. 